\title{
Poorer aerobic fitness relates to reduced integrity of multiple memory systems
}

\author{
Matthew B. Pontifex • Andrew C. Parks • Patrick C. O'Neil • \\ Adriel R. Egner • Joseph T. Warning • Karin A. Pfeiffer • \\ Kimberly M. Fenn
}

Published online: 4 March 2014

(C) Psychonomic Society, Inc. 2014

\begin{abstract}
Epidemiological investigations have revealed increases in the prevalence of sedentary behaviors in industrialized societies. However, the implications of those lifestyle choices and related cardiorespiratory fitness levels for memory function are not well-understood. To determine the extent to which cardiorespiratory fitness relates to the integrity of multiple memory systems, a cross-sectional sample of young adults were tested over the course of 3 days in areas related to implicit memory, working memory, long-term memory, and aerobic fitness. Findings revealed an association between aerobic fitness and memory function such that individuals with lower cardiorespiratory fitness exhibited poorer implicit memory performance and poorer long-term memory retention. These data indicate that cardiorespiratory fitness may be important for the optimal function of neural networks underlying these memory systems.
\end{abstract}

Keywords Basal ganglia · Cognitive control · Neural network · Working memory $\cdot$ Longterm memory $\cdot$ Implicit memory

Over the past several decades, individuals within industrialized societies have begun to lessen their engagement in physically active lifestyles while increasing consumption of energy-dense foods (Department of Health and Human Services [DHHS] and Department of Education [DOE], 2000). Accordingly, epidemiological investigations have suggested that a reduction in energy expenditure coupled with increased caloric consumption has resulted in an increase in the prevalence of several diseases, such

M. B. Pontifex • A. C. Parks • P. C. O’Neil • A. R. Egner •

J. T. Warning $\cdot$ K. A. Pfeiffer $\cdot$ K. M. Fenn

Michigan State University, East Lansing, MI, USA

M. B. Pontifex $(\bowtie)$

Department of Kinesiology, 27P IM Sports Circle, Michigan State

University, East Lansing, MI 48824-1049, USA

e-mail: pontifex@msu.edu as obesity, cardiovascular disease, colon cancer, and type-2 diabetes (DHHS and DOE, 2000). Such diseases have also been found to be independently related to aerobic fitness (i.e., the ability to sustain aerobic physical activities; DHHS, 2008). Yet beyond these effects on physical health, a growing body of research has begun to elucidate the detrimental effects that a lack of chronic physical activity and decreased aerobic fitness has on brain health and cognition (see Hillman, Erickson, \& Kramer, 2008, for a review).

Indeed, converging evidence across both human and animal models suggests that high-level cognitive systems such as memory may be sensitive to levels of aerobic fitness and physical activity. In rodent models, wheel-running has been associated with increases in neurogenesis in the dentate gyrus of the hippocampus (van Praag, Kempermann, \& Gage, 1999) and enhancements in hippocampal-dependent learning and memory processes (Vaynman, Ying, \& Gomez-Pinilla, 2004). Similarly, research in both preadolescent children and older adults has shown that poorer aerobic fitness relates to lower relational memory performance and smaller hippocampal volume (Chaddock et al., 2010a; Erickson et al., 2009). Indeed, aerobic exercise interventions have resulted in improvements in hippocampaldependent relational memory in preadolescent children (Monti, Hillman, \& Cohen, 2012) and reversal of age-related hippocampal volume loss in older adults (Erickson et al., 2011). More broadly, Smith and colleagues (2010) conducted a meta-analytic review of randomized controlled trials suggesting a modest relationship (Hedges's $g=0.128$ ) between aerobic exercise and declarative memory assessed using a wide range of tasks. A vast body of literature suggests that formation and retention of declarative memory is dependent on the hippocampus (see Squire, 1992, for a review). Indeed, recent evidence suggests that memory for declarative information is consolidated (i.e., is strengthened and shows increased resistance to interference or decay; McGaugh, 2000) after initial acquisition, potentially through a process of neuronal reactivation of information during waking or 
sleep (Carr, Jadhav, \& Frank, 2011; Dave \& Margoliash, 2000; Ji \& Wilson, 2007; Louie \& Wilson, 2001; Wilson \& McNaughton, 1994). Although there is strong evidence for a relationship between cardiovascular fitness and declarative memory ability, the vast majority of these investigations have focused on older adult populations (Smith et al., 2010), where it is believed that the influences of aerobic fitness should manifest most strongly as a result of age-related tissue loss. However, this epidemic of sedentary behaviors has existed for well over 2 decades. It therefore stands to reason that an association between aerobic fitness and declarative memory might also be observed in college-aged young adults who may have amassed a lifetime of relatively sedentary behavior. Accordingly, an initial aim of this study was to examine the relation between aerobic fitness and long-term declarative memory in a young-adult population. Given that exercise has been shown to enhance hippocampal function, we hypothesized that there would also be poorer offline processing of declarative memory in lower-fit individuals. Thus, young adults with lower aerobic fitness levels should exhibit greater loss of information over a retention period in a long-term memory task.

A secondary purpose of this investigation was to elucidate the extent to which the relationship between aerobic fitness and memory may generalize across multiple memory systems. There is some evidence to suggest that not all forms of memory are affected by fitness levels. For example, Smith and colleagues (2010) failed to observe a relationship between aerobic exercise interventions and working memory in older adults (Hedges's $g=0.03$ ). Working memory is considered to be a core component of high-level cognitive processes generally referred to as cognitive control (Davidson, Amso, Anderson, \& Diamond, 2006). Thus, these findings are counterintuitive, since a sizable body of literature in pediatric and older adult populations has shown the largest performance differentials for aspects of cognitive control, relative to other cognitive processes, in association with aerobic fitness (Hillman, Buck, Themanson, Pontifex, \& Castelli, 2009; Kramer, Colcombe, McAuley, Scalf, \& Erickson, 2005; Kramer et al., 1999; Pontifex et al., 2011). One potential interpretation for this discrepancy may be that fitness influences performance on working memory tasks that rely more on the manipulation or revision of information but may not influence performance on tasks that focus more on the maintenance of information. Accordingly, the extent to which working memory tasks differentially engage the maintenance and manipulation/revision components of working memory (Veltman, Rombouts, \& Dolan, 2003) may be critical to understanding the influence of aerobic fitness. Consonant with such an assertion, research in older adults (Gordon et al., 2008; Kramer et al., 2001) and women with chronic fatigue syndrome (Ickmans et al., 2013) has failed to observe a relationship between aerobic fitness and performance on the operation span and backward/forward digit span working memory tasks, which emphasize maintenance of information. In contrast, a 9-month randomized controlled intervention aimed at improving aerobic fitness in preadolescent children observed increased performance on a modified version of the Sternberg working memory task for the intervention group, relative to the control group (Kamijo et al., 2011). Similarly, Erickson et al. (2013) found that middle-aged (30 to 54 years) adults who engaged in less physical activity exhibited poorer performance on both letter and spatial variants of the $n$-back working memory tasks - which require the regular revision of information - than on their more active counterparts. Accordingly, an additional aim of this investigation was to provide insight into the extent to which aerobic fitness might differentially relate to these aspects of working memory. It was hypothesized that consonant with prior research, poorer aerobic fitness would relate to poorer performance on the $n$-back working memory task but would be unrelated to performance on working memory span tasks.

Previous research has also observed a relationship between aerobic fitness and neural regions associated with working memory. Indeed, research in both preadolescent children (Chaddock et al., 2010b) and older adults (Verstynen et al., 2012) has observed a relationship between aerobic fitness and the volume of the dorsal striatum, with lower fitness levels relating to decreased volume. Aerobic fitness has also been observed to relate to greater integrity of the prefrontal cortices in older adults (Colcombe et al., 2004). Furthermore, decreased activity within the prefrontal and parietal cortices has been observed for lower-fit, relative to higher-fit, individuals in both preadolescent children (Chaddock et al., 2012) and older adults (Colcombe et al., 2004). Although these regions heavily subserve working memory processes (Braver et al., 1997; MacDonald, Cohen, Stenger, \& Carter, 2000), these regions are also implicated in implicit memory (Grafton, Hazeltine, \& Ivry, 1995; Rauch et al., 1995; Robertson, Tormos, Maeda, \& Pascual-Leone, 2001; Torriero, Oliveri, Koch, Caltagirone, \& Petrosini, 2004; Willingham \& Koroshetz, 1993). Although little work has investigated the extent to which aerobic fitness might influence other aspects of human memory such as implicit memory, given that the neural regions underlying implicit memory are affected by fitness, it is logical to assume that fitness levels would affect implicit memory formation. Indeed, Quaney and colleagues (2009) observed improvements in implicit memory over the course of an 8-week exercise intervention in older adults. Although the extent to which aerobic fitness might relate to implicit memory in young adults is unknown, college-aged young adults who engage in relatively low levels of physical activity demonstrate poorer spatial priming, suggesting that they may have reduced implicit memory acquisition (Kamijo \& Takeda, 2009). Accordingly, the final aim of this investigation was to assess the relationship between aerobic fitness and implicit memory. We hypothesized that 
poorer aerobic fitness would be associated with poorer implicit memory. Collectively, the novel contribution of this investigation is the examination of the relationship between aerobic fitness and the integrity of multiple memory systems spanning long-term memory, working memory, and implicit memory, in a high-functioning young-adult population.

\section{Method}

Participants

Eighty-eight undergraduate students were recruited from Michigan State University. All participants provided prior written informed consent in accordance with the Michigan State University Institutional Review Board. All participants had scores above 90 on the Kaufman Brief Intelligence Test (K-BIT; Kaufman \& Kaufman, 1990), which was used to screen for the presence of cognitive abnormalities. Additionally, participants completed a health history and demographics questionnaire, reported being free of any neurological diseases or physical disabilities, and indicated normal or corrected-tonormal vision. Analyses were conducted on a final sample of 75 participants after excluding those participants who (1) did not have data across all of the cognitive assessments $(n=6)$, (2) achieved less than $50 \%$ accuracy in the $n$-back test $(n=6)$, and (3) achieved less than $90 \%$ accuracy in the serial reaction time task to ensure the integrity of this task as an index of implicit memory ( $n=1$; Curran, 1997). The racial and ethnic distribution of the sample was: 57 White or Caucasian, 11 Black or African American, 3 Asian, 1 Native Hawaiian or Other Pacific Islander, 3 biracial or of other ethnicities, with 3 identified as being of Hispanic origin. Demographic and fitness data for all participants are provided in Table 1.

Table 1 Participant demographic and fitness characteristics $( \pm S D)$

\begin{tabular}{ll}
\hline Measure & All Participants \\
\hline$N$ & 75 (40 females) \\
Age (years) & $20.2 \pm 2.2$ (minimum-maximum: $18-30)$ \\
Education (years) & $13.1 \pm 1.2$ (minimum-maximum: $12-17)$ \\
K-BIT composite (IQ) & $105.8 \pm 5.6$ (minimum-maximum: 92-120) \\
Body mass index & $23.2 \pm 3.4$ (minimum-maximum: $17.0-37.7)$ \\
Percentage of body fat & $18.6 \pm 7.7$ (minimum-maximum: $2.2-41.7)$ \\
$\mathrm{VO}_{2}$ max (ml/kg/min) & $44.4 \pm 8.6$ (minimum-maximum: 23.2-62.6) \\
$\mathrm{VO}_{2}$ max percentile & $52.1 \pm 33.5$ (minimum-maximum: 3-97) \\
\hline
\end{tabular}

Note. $\mathrm{VO}_{2} \max$ percentile is based on normative values for $\mathrm{VO}_{2} \max$ (Shvartz \& Reibold, 1990)
Test of memory systems

Five cognitive assessments were utilized to probe three different memory systems (long-term memory, working memory, and implicit memory), across three separate sessions. Long-term memory was tested over the span between the first and second sessions. Working memory was tested during the second (operation span [OSPAN] and symmetry span) and third ( $n$-back) sessions. Finally, implicit memory was tested during the third session.

\section{Long-term memory}

We assessed long-term memory over the course of two separate sessions, separated by $24 \mathrm{~h}$. During the first session, participants studied 68 semantically related word pairs (adapted from Fenn \& Hambrick, 2012). Word pairs were randomly displayed $\left(1.3^{\circ}\right.$ vertical visual angle $)$ at the center of the screen. Each pair was presented for 3,500 ms, with a 1,000 -ms intertrial interval. Immediately after training, participants were given a cued recall test on 60 word pairs. The first and final 4 pairs were not presented during the test, to control for primacy and recency effects on memory performance. During the test, the first word of each pair was presented at the center of the computer screen, and participants were asked to type the second word in a box directly below the first word of the pair. There was no time limit to respond. After each response, participants were first told whether their response was correct or incorrect and were then shown the correct word pair, regardless of response. Words were presented randomly during the test. Participants were trained to a criterion of $33 \%$ correct. After criterion was achieved, participants were given a final cued-recall test without feedback. During the second session ( $24 \mathrm{~h}$ following the first session), participants were again given a cued recall test on 60 word pairs, without feedback (see Fig. 1a for task example). Long-term memory performance was quantified by calculating the number of paired-associate items that were lost across the 24-h retention interval, corrected by the total number of items recalled in the final test during session 1 [(items lost/total items recalled in session 1) $\times 100$ ]. Thus, lower values indicated better memory retention. This approach ensured that any observed effects could not be explained by greater loss for individuals who demonstrated better performance during session 1 .

\section{Working memory}

We assessed working memory using the OSPAN task (Unsworth, Heitz, Schrock, \& Engle, 2005), the symmetry span task (Kane et al., 2004), and a spatial $n$-back task (1back; Drollette, Shishido, Pontifex, \& Hillman, 2012). These tasks were chosen because they allowed us to investigate the 

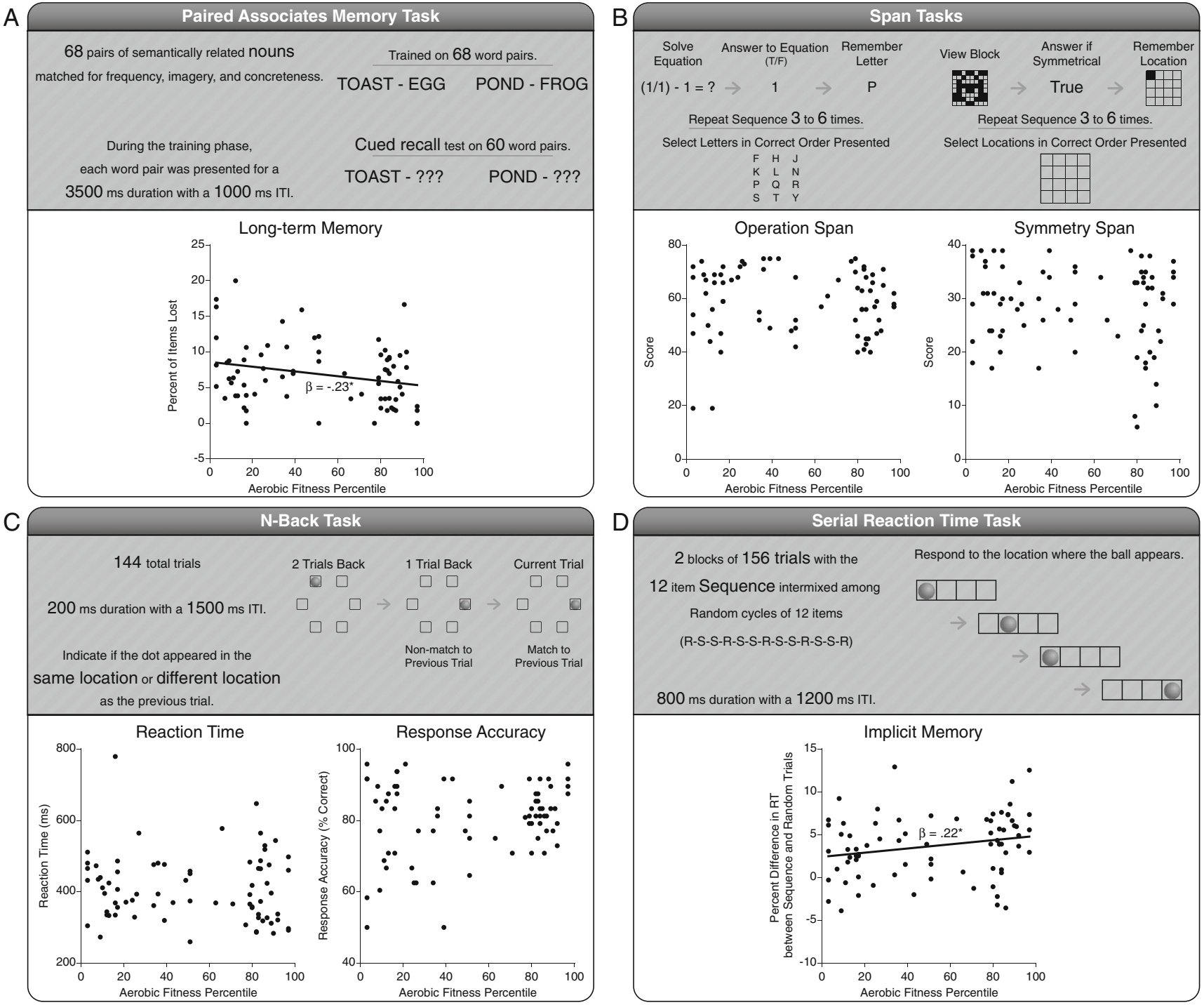

Fig. 1 Graphic representation of the long-term memory task (a), the operation/symmetry span tasks (b), the $n$-back task (c), and the serial reaction time task (d) with their respective relationships relative to aerobic fitness percentile

extent to which aerobic fitness may differentially influence two components of working memory, maintenance and manipulation (Veltman et al., 2003).

In the OSPAN task (completed during the second session), participants were asked to maintain verbal information in memory while performing arithmetic problems. On each item, participants were first given an arithmetic problem and were then given a potential solution to the problem and had to verify whether the solution was correct or incorrect. They responded by clicking on "True" if the solution was correct and clicking on the word "False" if the solution was not correct. After each problem, they were given a letter $\left(2^{\circ}\right.$ vertical visual angle) to remember (from the set: $\mathrm{F}, \mathrm{H}, \mathrm{J}, \mathrm{K}$, $\mathrm{L}, \mathrm{N}, \mathrm{P}, \mathrm{Q}, \mathrm{R}, \mathrm{S}, \mathrm{T}, \mathrm{Y})$. After between three and seven problems and letters, they were asked to recall the letters in the order in which they were presented. During the recall phase, the 12 letters appeared in a grid on the screen $\left(12^{\circ}\right.$ vertical visual angle), and participants clicked on the letters, in the order in which they were presented. After the letter recall phase, they were given feedback on the number of letters that were correctly recalled and the number of math errors for that set of trials (see Fig. 1b for task example; Unsworth et al., 2005). Prior to the start of the task, participants were given 4 practice trials on letter recall and 15 practice trials on the arithmetic trials separately, followed by 3 practice trials with the letter recall and arithmetic trials presented in the same fashion as in the actual task. Participants then completed 3 trials of each set size $(3,4,5,6$, and 7 letters) and were asked to remember 75 letters amid a total of 75 math problems. In the symmetry span task (completed during the second session), participants were asked to maintain spatial information in memory while performing a spatial judgment. On each item, participants were presented with an $8 \times 8$ grid $\left(12^{\circ}\right.$ vertical visual angle) with some of the squares filled in with 
black and were asked to judge whether the geometrical image was symmetrical. They were then shown a $4 \times 4$ grid $\left(12^{\circ}\right.$ vertical visual angle) with one red square filled in and were asked to remember the location of the filled square. After between two and five items (symmetry judgment + spatial memory), participants were asked to recall the location of the red squares that they held in memory in the order in which they appeared (see Fig. 1b; Kane et al., 2004). There were a total of 12 trials in the experiment. Prior to test trials, participants completed 3 practice memory trials and 14 practice symmetry judgments. They also completed 3 practice trials in which they did both symmetry judgments and spatial memory tasks. Thus, in both the OSPAN and symmetry span tasks, participants performed a secondary processing task (i.e., arithmetic or symmetry judgment) while maintaining information within working memory (i.e., letters or locations).

The $n$-back task (completed during the third session) places a continuous load on working memory. Participants were required to update and reorganize memory representations as a red dot moved pseudorandomly among six fixed spatial locations $\left(1.7^{\circ}\right.$ horizontal and vertical visual angles) spaced in a circular fashion on a $4.3^{\circ}$ radius from the center of the screen (Drollette, Shishido, Pontifex, \& Hillman, 2012). Participants were instructed to respond as quickly and as accurately as possible to indicate whether the red $\operatorname{dot}\left(0.7^{\circ}\right.$ radius $)$ appeared in the same spatial location ( $33.3 \%$ of the time) or a different spatial location as on the previous trial. Thus, in order to recall both the location and the sequence of the stimuli, the $n$-back task requires the regular revision of working memory as each new stimulus replaces the previous to become the new matching location (see Fig. 1c; Chen, Mitra, \& Schlaghecken, 2008). Prior to the start of each condition, participants were given blocks of 40 practice trials until they achieved a minimum $50 \%$ accuracy. Participants completed a block of 144 trials with stimuli presented for $200 \mathrm{~ms}$ and a $1,500 \mathrm{~ms}$ intertrial interval.

\section{Implicit memory}

We assessed implicit procedural memory (completed during the third session) using the serial reaction time task (SRTT) which is a four-choice reaction time task embedded with a repeating sequence (see Fig. 1d for task example; Robertson, 2007). This sequence consisted of a 12 -stimulus run (1-2-14-2-3-4-3-1-4-2-3) in which each element provides probabilistically predictive information (67\% of the time) about the next element in the sequence. Participants were instructed to respond to the location of the stimulus $\left(0.4^{\circ}\right.$ radius red dot within a field of four boxes arranged horizontally, each with $2^{\circ}$ horizontal and vertical visual angles; see Fig. 1d) as quickly and as accurately as possible using a response pad with buttons mapped to the index and middle fingers on each hand. Following completion of 40 practice trials, participants completed two blocks of 156 trials with the 12-item sequence (S) intermixed among random $(\mathrm{R})$ cycles of 12 trials (i.e., R-S-SR-S-S-R-S-S-R-S-S-R; Curran, 1997). Implicit memory performance was quantified as the difference in reaction time between sequence and random trials relative to the random sequence trial reaction time ([(sequence trials - random trials)/ random trials $] \times 100$ ), with larger values indicating superior implicit memory. Stimuli were presented for $800 \mathrm{~ms}$, with a 1,200-ms intertrial interval.

Fitness and body composition assessment

Cardiorespiratory fitness was assessed using a test of maximal oxygen consumption $\left(\mathrm{VO}_{2} \mathrm{max}\right)$, which describes the physiological limit to the rate at which an individual can deliver/ consume oxygen and is considered the criterion measure of cardiorespiratory fitness (American College of Sports Medicine, 2006). Relative peak oxygen consumption (ml/kg/ $\mathrm{min}$ ) was measured using a computerized indirect calorimetry system (ParvoMedics True Max 2400) while participants ran or walked on a motor-driven treadmill at a constant speed, with incremental increases of $2.5 \%$ grade every 2 min until the participant was no longer able to maintain the exercise intensity (American College of Sports Medicine, 2006). Attainment of maximal effort was evidenced by attainment of two of the following four criteria: (1) a plateau in oxygen consumption corresponding to an increase of less than $2 \mathrm{ml} /$ $\mathrm{kg} / \mathrm{min}$ despite an increase in workload; (2) a peak heart rate within 10 beats per minute of age-predicted maximum (i.e., 220 -age); (3) respiratory exchange ratio $\geq 1.1$; or (4) OMNI perceived exertion scale rating $>7$ (Pontifex, Hillman, \& Polich, 2009). Cardiorespiratory fitness percentiles were extracted from normative data provided by Shvartz and Reibold (1990). Weight and percent body fat were quantified using an Omron full-body composition monitor (Omron Healthcare, Inc. HBF-510 W).

\section{Statistical analysis}

Prior to analysis, all study variables were screened for homoscedasticity and normality. Bivariate correlation analyses were then conducted using multiple probability corrected Pearson product-moment correlation coefficients (Curtin \& Schulz, 1998) between demographic factors and each memory domain. Hierarchical linear regression analyses were then performed to explain variance in these factors as they related to aerobic fitness. This was undertaken by regressing memory domains on descriptive factors (i.e., age, sex, race, IQ, and percentage of body fat) that were statistically significant correlates in step 1 to judge the independent contribution of aerobic fitness (as assessed using $\mathrm{VO}_{2}$ max percentile) in step 2 for explaining variance beyond that of the descriptive variables (Kamijo et al., 2012). All data analyses were performed in PASW Statistics, 20.0 (IBM, Somers, NY) utilizing a familywise alpha 
level of $p=.05$. Overall means and variability measures are provided for each memory domain in Table 2. Statistical summaries of the correlational and regression analyses for each memory domain are provided in Tables 3 and 4, respectively.

\section{Results}

Long-term memory task

Hierarchical regression analysis indicated that individuals with lower-fitness exhibited less stable memory as indexed by a greater proportion of items lost between session 1 and session $2, \beta=-0.23, t(71)=2.2, p=.03$ (see Fig. 1a), even after controlling for age, sex, and IQ. ${ }^{1}$

\section{Working memory tasks}

No relationships with fitness were observed for the OSPAN task, symmetry span task, or $n$-back task, $|r s| \leq .19, p \mathrm{~s} \geq .11$.

Implicit memory task

Findings revealed that individuals with poorer aerobic fitness exhibited inferior implicit memory acquisition, $\beta=0.22, t(73)$ $=1.95, p=.05$ (see Fig. 1d).

\section{Discussion}

The aim of the present investigation was to examine the relationship between aerobic fitness and the integrity of multiple memory systems. Consistent with prior research, we found a relationship between fitness and declarative memory; individuals with lower levels of aerobic fitness demonstrated less stable long-term memory. We also found that aerobic fitness was related to implicit memory in college-aged young adults. Finally, one of the goals of this investigation was to examine the extent to which the type of working memory task

\footnotetext{
${ }^{11}$ Accelerometry was also used to assess levels of physical activity over the time period between memory acquisition (session 1) and retrieval (session 2) in the long-term memory task to ensure that any observed effects were not the result of acute bouts of physical activity, rather than cardiorespiratory fitness. Participants were given an (Actigraph, GT3X) accelerometer at the end of the day 1 session and were instructed to wear the accelerometer for the following 24-h period (excluding during sleep and shower times). The participants were shown how to position the device, so that all participants wore the accelerometer on their right hip. Data were recorded in 1-s epoch lengths and reintegrated into 60-s epochs for analysis using ActiLife 6 data analysis software. The accelerometer cutpoints as established by Freedson (Freedson, Melanson, \& Sirard, 1998) determined physical activity intensity. No significant relationship was observed between any index of moderate or higher intensity physical activity over the 24 - $\mathrm{h}$ period between sessions and long-term memory, $|r \mathrm{~s}|$ $\leq .11, p \mathrm{~s} \geq .39$
}

Table 2 Mean $( \pm S D)$ performance across each memory domain

\begin{tabular}{ll}
\hline Measure & All Participants \\
\hline Long-term memory (\%) & $6.9 \pm 4.4$ \\
Working memory & \\
Operation span & $58.8 \pm 12.5$ \\
Symmetry span & $28.5 \pm 7.8$ \\
$N$-back target reaction time (ms) & $409.4 \pm 93.4$ \\
$N$-back target accuracy (\% correct) & $80.4 \pm 10.5$ \\
Implicit memory (\% lost) & $3.7 \pm 3.7$ \\
\hline
\end{tabular}

and the degree to which the task differentially engages the maintenance and manipulation components of working memory might explain the inconsistent evidence in the literature of a relationship between aerobic fitness and working memory. However, contrary to our hypothesis, no relationship between aerobic fitness and any of our three indices of working memory were observed.

Accordingly, the present findings appear consistent with those reported in a recent meta-analysis of randomized controlled trials that failed to observe a relationship between fitness and memory span based indices of working memory (Smith et al., 2010). However, in contrast to findings reported by Kamijo and colleagues (2011) and Erickson and colleagues (2013), we did not find a relationship between aerobic fitness and working memory tasks that emphasize manipulation or revision of information. Such discrepant findings may be a function of a number of factors. In particular, much of the prior research has investigated the association between aerobic fitness/physical activity and working memory in older adult populations, but our investigation utilized a sample of college-aged adults. The high-functioning nature of this population may limit the extent to which fitness-related differences in performance are able to be observed. Thus, future research is still necessary to investigate the extent to which the maintenance and manipulation of information within working memory may differentially relate to aerobic fitness during development and aging. It is also important to note that the findings reported by Kamijo et al. (2011) and Erickson et al. (2013) are methodologically more rigorous than the present investigation, employing a longitudinal randomized controlled trial and a sample of over a thousand adults, respectively. Accordingly, it may be that the relationship between fitness and these components of working memory is sufficiently small to necessitate randomized controlled trials or much larger sample sizes in cross-sectional designs than were employed by the present investigation to be detected.

Within the physical activity/cardiorespiratory fitness and cognition literature, cognitive reserve theory (Stern, 2002) has often been posited as an explanation for the relationship between health behaviors and attributes and cognitive function, but we do not believe that it can adequately explain our 
Table 3 Bivariate correlations between demographic factors and memory domains

\begin{tabular}{|c|c|c|c|c|c|c|}
\hline Variable & Age & $\begin{array}{l}\text { Sex }(0=\text { Female, } \\
1=\text { Male })\end{array}$ & $\begin{array}{l}\text { Race }(0=\text { White, } \\
1=\text { Nonwhite })\end{array}$ & $\begin{array}{l}\text { K-BIT } \\
\text { Composite (IQ) }\end{array}$ & $\begin{array}{l}\text { Percentage of } \\
\text { Body Fat }\end{array}$ & $\begin{array}{l}\text { Aerobic Fitness } \\
\left(\mathrm{VO}_{2} \mathrm{max} \text { Percentile }\right)\end{array}$ \\
\hline Long-term memory & $.28 *$ & $.26^{* *}$ & .12 & $-.33^{* *}$ & .01 & $-.25^{*}$ \\
\hline \multicolumn{7}{|l|}{ Working memory } \\
\hline Operation span & -.17 & -.14 & .07 & .18 & -.23 & .03 \\
\hline Symmetry span & .01 & .04 & .20 & $.25^{*}$ & -.04 & -.14 \\
\hline$N$-back target reaction time & -.11 & -.21 & $.27 * *$ & -.06 & .03 & -.08 \\
\hline$N$-back target accuracy $(\%$ correct $)$ & .21 & .04 & -.04 & .12 & .01 & .19 \\
\hline Implicit memory & -.08 & -.08 & .02 & -.09 & -.14 & $.22 *$ \\
\hline
\end{tabular}

${ }^{*} p \leq .05$

$* * p \leq .025$

results. In elderly adults and preadolescent children, these cognitive reserves are believed to be reduced, resulting in lower-fit individuals exhibiting a decreased ability to respond to high-level task demands (Hillman et al., 2008; Pontifex et al., 2011). Speculatively, however, our findings appear to suggest that the relationship between fitness and cognition may not be explained by the extent to which a particular cognitive process can flexibly respond to increasing demands but, rather, may depend on the specific neural networks that underlie the cognitive processes. That is, within the present investigation, we observed a relationship between fitness and implicit memory using a serial reaction time task. This task (responding to a simple four-choice reaction time task) places relatively low demands on cognitive processing. Thus, the extent to which this task would sufficiently tax these processes to necessitate cognitive reserve is questionable. However, previous research has shown that this form of implicit memory is heavily mediated by the basal ganglia, cerebellum, and prefrontal cortex (Grafton et al., 1995; Rauch et al., 1995; Robertson et al., 2001; Torriero et al.,2004; Willingham \& Koroshetz, 1993). These structures have previously been shown to exhibit a relationship with aerobic fitness, with lower-fit preadolescent children and older adults exhibiting smaller dorsal striatum volume of the basal ganglia (Chaddock et al., 2010b; Verstynen et al., 2012) and decreased activity of prefrontal and parietal cortices (Chaddock et al., 2012; Colcombe et al., 2004). Thus, the apparent association between implicit memory and aerobic fitness may be due to a direct effect of exercise on the neural structures that support this task.

This neural network hypothesis would also support the finding of an association between lower aerobic fitness and increased memory loss in the paired-associates memory task. Specifically, long-term declarative memory tasks have previously been shown to be heavily dependent upon the hippocampus (Squire, 1992), and aerobic fitness has been found to relate to hippocampal volume in rodents (Pereira et al., 2007), children (Chaddock et al., 2010a), and older adults (Erickson et al., 2009; Erickson et al., 2011). Wheel running in rodents has been found to result in increases in hippocampal cell proliferation and survival (van Praag et al., 1999), growth factors (Cotman \& Berchtold, 2002), dendritic structure (Redila \& Christie, 2006), and gliogenesis (Uda, Ishido, Kami, \& Masuhara, 2006). However, it is also possible that

Table 4 Summary of hierarchical regression analyses for the relationship between fitness and memory domains

\begin{tabular}{|c|c|c|c|c|c|c|c|}
\hline & $R^{2}$ & $R^{2}$ Change & $F$ Change & $B$ & $S E B$ & $\beta$ & $t$ \\
\hline Long-term memory & .23 & $.05^{*}$ & 4.82 & 0.00 & 0.00 & -0.23 & $2.20^{*}$ \\
\hline \multicolumn{8}{|l|}{ Working memory } \\
\hline Operation span & .001 & .001 & 0.08 & -0.01 & 0.04 & -0.03 & 0.28 \\
\hline Symmetry span & .02 & .02 & 1.41 & -0.03 & 0.03 & -0.14 & 1.19 \\
\hline $\mathrm{N}$-back target reaction time & .006 & .006 & 0.44 & -0.21 & 0.33 & -0.08 & 0.66 \\
\hline$N$-back target accuracy $(\%$ correct $)$ & .04 & .04 & 2.64 & 0.06 & 0.04 & 0.19 & 1.63 \\
\hline Implicit memory & .05 & $.05^{*}$ & 3.82 & 0.00 & 0.00 & 0.22 & $1.95^{*}$ \\
\hline
\end{tabular}

Note. Model for long-term memory included age, sex, and IQ. No demographic predictors were identified for implicit memory or any measure of working memory

$* p \leq .05$

${ }^{\star}$ Relative to model of demographic factors 
aerobic fitness exerts indirect effects on long-term memory retention. Declarative memory is strengthened during sleep (for reviews, see Diekelmann \& Born, 2010; McGaugh, 2000) - in particular, during slow-wave sleep (SWS; Plihal $\&$ Born, 1997). Given previous research indicating that exercise increases SWS (see Driver \& Taylor, 2000, for a review), lower-fit individuals may obtain poorer quality sleep resulting in decreased memory consolidation during sleep. Although investigating the precise relationship between fitness, sleep, and long-term memory was beyond the scope of the present investigation, future research should investigate the specificity of the effect of exercise on long-term memory and should include moderating variables, such as sleep quality.

Although we propose that the present results are most parsimoniously explained by the neural network hypothesis, it is important to note that this hypothesis is not intrinsically at odds with the cognitive reserve perspective. Rather, a decreased ability to flexibly modulate cognitive operations and decreased efficiency of these systems would be expected if a lack of aerobic fitness contributes to degradation of the specific neural networks that support high-level cognitive operations. A key limitation of the present investigation and much of the work in this area, however, is their cross-sectional nature. Thus, longitudinal randomized controlled trials are still necessary to determine the extent to which these relationships are a function of aerobic fitness or are simply a product of other individual-difference factors. This is particularly evident since, although an association between fitness and striatal volume has been observed cross-sectionally (Chaddock et al., 2010b; Verstynen et al., 2012); a year-long randomized controlled physical activity intervention in older adults failed to observe any changes in the caudate nucleaus (Erickson et al., 2011). Accordingly, since neuroimaging modalities are increasingly utilized within this area of study, an important question will be the extent to which physical-activity-induced changes in aerobic fitness differentially influence these underlying brain structures and networks throughout the lifespan. Examination of the dose-response curve for these neural regions that appear to be sensitive to differences in aerobic fitness may also elucidate some of these apparently discrepent findings between cross-sectional and longitudinal research. Although Erickson and colleagues (2011) did not observe any statistical differences in the caudate nucleaus associated with their physical activity intervention in older adults, a slight positive trend is apparent. Thus, while some neural regions might respond relatively quickly to physical activity (i.e., over the course of a 1 -year intervention), others might exhibit a more protracted response or may require more frequent/ intense bouts of activity or activity of a different type to incur any changes. Collectively, however, these findings contribute to a larger body of research that has begun to indicate that cardiorespiratory fitness may be important for the optimal functioning of multiple aspects of high-level cognitive and memory processes.

Author Disclosure Statement No conflicting financial interests exist.

\section{References}

American College of Sports Medicine. (2006). ACSM's guidelines for exercise testing and prescription (7th ed.). New York: Lippincott Williams \& Wilkins.

Braver, T. S., Cohen, J. D., Nystrom, L. E., Jonides, J., Smith, E. E., \& Noll, D. C. (1997). A parametric study of prefrontal cortex involvement in human working memory. NeuroImage, 5, 49-62. doi:10. 1006/nimg.1996.0247

Carr, M. F., Jadhav, S. P., \& Frank, L. M. (2011). Hippocampal replay in the awake state: A potential substrate for memory consolidation and retrieval. Nature Neuroscience, 14, 147-153. doi:10.1038/nn.2732

Chaddock, L., Erickson, K. I., Prakash, R. S., Kim, J. S., Voss, M. W., VanPatter, M., ... Kramer, A. F. (2010a). A neuroimaging investigation of the association between aerobic fitness, hippocampal volume, and memory performance in preadolescent children. Brain Research, 1358, 172-183. doi:10.1016/j.brainres.2010.08.049

Chaddock, L., Erickson, K. I., Prakash, R. S., VanPatter, M., Voss, M. W., Pontifex, M. B., ... Kramer, A. F. (2010b). Basal ganglia volume is associated with aerobic fitness in preadolescent children. Developmental Neuroscience, 32, 249-256. doi:10.1159/000316648

Chaddock, L., Erickson, K. I., Prakash, R. S., Voss, M. W., VanPatter, M., Pontifex, M. B., . . . Kramer, A. F. (2012). A functional MRI investigation of the association between childhood aerobic fitness and neurocognitive control. Biological Psychology, 89, 260-268. doi:10.1016/j.biopsycho.2011.10.017

Chen, Y. N., Mitra, S., \& Schlaghecken, F. (2008). Sub-processes of working memory in the N-back task: An investigation using ERPs. Clinical Neurophysiology, 119, 1546-1559. doi:10.1016/j. clinph.2008.03.003

Colcombe, S. J., Kramer, A. F., Erickson, K. I., Scalf, P., McAuley, E., Cohen, N. J., ... Elavsky, S. (2004). Cardiovascular fitness, cortical plasticity, and aging. Proceedings of the National Academy of Sciences of the United States of America, 101, 3316-3321. doi:10. 1073/pnas.0400266101

Cotman, C. W., \& Berchtold, N. C. (2002). Exercise: A behavioral intervention to enhance brain health and plasticity. Trends in Neurosciences, 25, 295-301. doi:10.1016/S0166-2236(02)02143-4

Curran, T. (1997). Higher-order associative learning in amnesia: Evidence from the serial reaction time task. Journal of Cognitive Neuroscience, 9, 522-533. doi:10.1162/jocn.1997.9.4.522

Curtin, F., \& Schulz, P. (1998). Multiple correlations and bonferroni's correction. Biological Psychiatry, 44, 775-777. doi:10.1016/S00063223(98)00043-2

Dave, A. S., \& Margoliash, D. (2000). Song replay during sleep and computational rules for sensorimotor vocal learning. Science, 290 , 812-816. doi:10.1126/science.290.5492.812

Davidson, M. C., Amso, D., Anderson, L. C., \& Diamond, A. (2006). Development of cognitive control and executive functions from 4 to 13 years: Evidence from manipulations of memory, inhibition, and task switching. Neuropsychologia, 44, 2037-2078. doi:10.1016/j. neuropsychologia.2006.02.006 
Department of Health and Human Services. (2008). 2008 Physical Activity Guidelines for Americans (ODPHP Publication No. U0036). Washington, DC: Government Printing Office.

Department of Health and Human Services [DHHS] and Department of Education [DOE]. (2000). Promoting better health for young people through physical activity and sports. A report to the President from the Secretary of Health and Human Services and the Secretary of Education. Silver Spring, MD: Centers for Disease Control.

Diekelmann, S., \& Born, J. (2010). The memory function of sleep. Nature Reviews Neuroscience, 11, 114-126. doi:10.1038/nrn2762

Driver, H. S., \& Taylor, S. R. (2000). Exercise and sleep. Sleep Medicine Reviews, 4, 387-402. doi:10.1053/smrv.2000.0110

Drollette, E. S., Shishido, T., Pontifex, M. B., \& Hillman, C. H. (2012). Maintenance of cognitive control during and after walking in preadolescent children. Medicine and Science in Sports and Exercise, 44, 2017-2024. doi:10.1249/MSS.0b013e318258bcd5

Erickson, K. I., Banducci, S. E., Weinstein, A. M., MacDonald, A. W., Ferrell, R. E., Halder, I., ... Manuck, S. B. (2013). The brain-derived neurotrophic factor Val66Met polymorphism moderates an effect of physical activity on working memory performance. Psychological Science, 24, 1770-1779. doi:10.1177/0956797613480367

Erickson, K. I., Prakash, R. S., Voss, M. W., Chaddock, L., Hu, L., Morris, K. S., ... Kramer, A. F. (2009). Aerobic fitness is associated with hippocampal volume in elderly humans. Hippocampus, 19, 1030-1039. doi:10.1002/hipo.20547

Erickson, K. I., Voss, M. W., Prakash, R. S., Basak, C., Szabo, A., Chaddock, L., ... Kramer, A. F. (2011). Exercise training increases size of hippocampus and improves memory. Proceedings of the National Academy of Sciences of the United States of America, 108, 3017-3022. doi:10.1073/pnas.1015950108

Fenn, K. M., \& Hambrick, D. Z. (2012). Individual differences in working memory capacity predict sleep-dependent memory consolidation. Journal of Experimental Psychology. General, 141, 404-410. doi:10.1037/a0025268

Freedson, P. S., Melanson, E., \& Sirard, J. (1998). Calibration of the computer science and applications, Inc. accelerometer. Medicine and Science in Sports and Exercise, 777-781.

Gordon, B. A., Rykhlevskaia, E. I., Brumback, C. R., Lee, Y., Elavsky, S., Konopack, J. F., ... Fabiani, M. (2008). Neuroanatomical correlates of aging, cardiopulmonary fitness level, and education. Psychophysiology, 45, 825-838. doi:10.1111/j.1469-8986.2008.00676.x

Grafton, S. T., Hazeltine, E., \& Ivry, R. (1995). Functional mapping of sequence learning in normal humans. Journal of Cognitive Neuroscience, 7, 497-510. doi:10.1162/jocn.1995.7.4.497

Hillman, C. H., Buck, S. M., Themanson, J. R., Pontifex, M. B., \& Castelli, D. M. (2009). Aerobic fitness and cognitive development: Event-related brain potential and task performance indices of executive control in preadolescent children. Developmental Psychology, 45, 114-129. doi:10.1037/a0014437

Hillman, C. H., Erickson, K. I., \& Kramer, A. F. (2008). Be smart, exercise your heart: Exercise effects on brain and cognition. Nature Reviews Neuroscience, 9, 58-65. doi:10.1038/nrn2298

Ickmans, K., Clarys, P., Nijs, J., Meeus, M., Aerenhouts, D., Zinzen, E., ... Pattyn, N. (2013). Association between cognitive performance, physical fitness, and physical activity level in women with chronic fatigue syndrome. Journal of Rehabilitation Research \& Development, 50, 795-810. doi:10.1682/JRRD.2012.08.0156

Ji, D., \& Wilson, M. A. (2007). Coordinated memory replay in the visual cortex and hippocampus during sleep. Nature Neuroscience, 10, 100-107. doi:10.1038/nn1825

Kamijo, K., Khan, N. A., Pontifex, M. B., Scudder, M. R., Drollette, E. S., Raine, L. B., ... Hillman, C. H. (2012). The relation of adiposity to cognitive control and scholastic achievement in preadolescent children. Obesity, 20, 2406-2411. doi:10.1038/oby.2012.112

Kamijo, K., Pontifex, M. B., O'Leary, K. C., Scudder, M. R., Wu, C.-T., Castelli, D. M., \& Hillman, C. H. (2011). The effects of an afterschool physical activity program on working memory in preadolescent children. Developmental Science, 14, 1046-1058. doi:10. 1111/j.1467-7687.2011.01054.x

Kamijo, K., \& Takeda, Y. (2009). General physical activity levels influence positive and negative priming effects in young adults. Clinical Neurophysiology, 120, 511-519. doi:10.1016/j.clinph.2008.11.022

Kane, M. J., Hambrick, D. Z., Tuholski, S. W., Wilhelm, O., Payne, T. W., \& Engle, R. W. (2004). The generality of working memory capacity: A latent-variable approach to verbal and visuospatial memory span and reasoning. Journal of Experimental Psychology. General, 133, 189-217. doi:10.1037/0096-3445.133.2.189

Kaufman, A. S., \& Kaufman, N. L. (1990). Kaufman brief intelligence test manual. Circle Pines, MN: American Guidance Service.

Kramer, A. F., Colcombe, S. J., McAuley, E., Scalf, P. E., \& Erickson, K. I. (2005). Fitness, aging and neurocognitive function. Neurobiology of Aging, 26, 124-127. doi:10.1016/j.neurobiolaging.2005.09.009

Kramer, A. F., Hahn, S., Cohen, N. J., Banich, M. T., McAuley, E., Harrison, C. R., ... Colcombe, A. (1999). Ageing, fitness and neurocognitive function. Nature, 400, 418-419. doi:10.1038/22682

Kramer, A. F., Hahn, S., McAuley, E., Cohen, N. J., Banich, M. T., Harrison, C., ... Vakil, E. (2001). Exercise, aging and cognition: Healthy body, healthy mind? In A. D. Fisk, \& W. Rogers (Eds.), Human Factors Interventions for the Health Care of Older Adults (pp. 91-120). Hillsdale, N.J.: Erlbaum.

Louie, K., \& Wilson, M. A. (2001). Temporally structured replay of awake hippocampal ensemble activity during rapid eye movement sleep. Neuron, 29, 145-156. doi:10.1016/S0896-6273(01)00186-6

MacDonald, A. W., Cohen, J. D., Stenger, V. A., \& Carter, C. S. (2000). Dissociating the role of the dorsolateral prefrontal and anterior cingulate cortex in cognitive control. Science, 288, 1835-1838. doi:10.1126/science. 288.5472 .1835

McGaugh, J. L. (2000). Memory - a century of consolidation. Science, 287, 248-251. doi:10.1126/science.287.5451.248

Monti, J. M., Hillman, C. H., \& Cohen, N. J. (2012). Aerobic fitness enhances relational memory in preadolescent children: The FITKids randomized control trial. Hippocampus, 22, 1876-1882. doi:10.1002/hipo.22023

Pereira, A. C., Huddleston, D. E., Brickman, A. M., Sosunov, A. A., Hen, R., McKhann, G. M., ... Small, S. A. (2007). An in vivo correlate of exercise-induced neurogenesis in the adult dentate gyrus. Proceedings of the National Academy of Sciences of the United States of America, 104, 5638-5643. doi:10.1073/pnas.0611721104

Plihal, W., \& Born, J. (1997). Effects of early and late nocturnal sleep on declarative and procedural memory. Journal of Cognitive Neuroscience, 9, 534-547. doi:10.1162/jocn.1997.9.4.534

Pontifex, M. B., Hillman, C. H., \& Polich, J. (2009). Age, physical fitness, and attention: P3a and P3b. Psychophysiology, 46, 379387. doi:10.1111/j.1469-8986.2008.00782.x

Pontifex, M. B., Raine, L. B., Johnson, C. R., Chaddock, L., Voss, M. W., Cohen, N. J., ... Hillman, C. H. (2011). Cardiorespiratory fitness and the flexible modulation of cognitive control in preadolescent children. Journal of Cognitive Neuroscience, 23, 1332-1345. doi:10. 1162 /jocn.2010.21528

Quaney, B. M., Boyd, L. A., McDowd, J. M., Zahner, L. H., He, J., Mayo, M. S., \& Macko, R. F. (2009). Aerobic exercise improves cognition and motor function poststroke. Neurorehabilitation and Neural Repair, 23, 879-885. doi:10.1177/1545968309338193

Rauch, S. L., Savage, C. R., Brown, H. D., Curran, T., Alpert, N. M., Kendrick, A., ... Kosslyn, S. M. (1995). A PET investigation of implicit and explicit sequence learning. Human Brain Mapping, 3, 271-286. doi: $10.1002 / \mathrm{hbm} .460030403$

Redila, V. A., \& Christie, B. R. (2006). Exercise-induced changes in dendritic structure and complexity in the adult hippocampal dentate gyrus. Neuroscience, 137, 1299-1307. doi:10.1016/j.neuroscience.2005.10.050

Robertson, E. M. (2007). The serial reaction time task: Implicit motor skill learning? The Journal of Neuroscience, 27, 10073-10075. doi: 10.1523/JNEUROSCI.2747-07.2007 
Robertson, E. M., Tormos, J. M., Maeda, F., \& Pascual-Leone, A. (2001). The role of the dorsolateral prefrontal cortex during sequence learning is specific for spatial information. Cerebral Cortex, 11, 628-635. doi:10.1093/cercor/11.7.628

Shvartz, E., \& Reibold, R. C. (1990). Aerobic fitness norms for males and females aged 6 to 75 years: A review. Aviation, Space, and Environmental Medicine, 61, 3-11.

Smith, P. J., Blumenthal, J. A., Hoffman, B. M., Cooper, H., Strauman, T. A., Welsh-Bohmer, K., ... Sherwood, A. (2010). Aerobic exercise and neurocognitive performance: A metaanalytic review of randomized controlled trials. Psychosomatic Medicine, 72, 239-252. doi:10.1097/PSY. 0b013e3181d14633

Squire, L. R. (1992). Memory and the hippocampus: A synthesis from findings with rats, monkeys, and humans. Psychological Review, 99, 195-231. doi:10.1037/0033-295X.99.2.195

Stern, Y. (2002). What is cognitive reserve? Theory and research application of the reserve concept. Journal of the International Neuropsychological Society, 8, 448-460.

Torriero, S., Oliveri, M., Koch, G., Caltagirone, C., \& Petrosini, L. (2004). Interference of left and right cerebellar rTMS with procedural learning. Journal of Cognitive Neuroscience, 16, $1605-1611$.

Uda, M., Ishido, M., Kami, K., \& Masuhara, M. (2006). Effects of chronic treadmill running on neurogenesis in the dentate gyrus of the hippocampus of adult rat. Brain Research, 1104, 64-72. doi:10. 1016/j.brainres.2006.05.066

Unsworth, N., Heitz, R. P., Schrock, J. C., \& Engle, R. W. (2005). An automated version of the operation span task. Behavior Research Methods, 37, 498-505. doi:10.3758/BF03192720

van Praag, H., Kempermann, G., \& Gage, F. H. (1999). Running increases cell proliferation and neurogenesis in the adult mouse dentate gyrus. Nature Neuroscience, 2, 266-270. doi:10.1038/6368

Vaynman, S., Ying, Z., \& Gomez-Pinilla, F. (2004). Hippocampal BDNF mediates the efficacy of exercise on synaptic plasticity and cognition. European Journal of Neuroscience, 20, 2580-2590. doi:10. $1111 / j .1460-9568.2004 .03720 . x$

Veltman, D. J., Rombouts, S. A., \& Dolan, R. J. (2003). Maintenance versus manipulation in verbal working memory revisited: An fMRI study. NeuroImage, 18, 247-256. doi:10.1016/S1053-8119(02)00049-6

Verstynen, T. D., Lynch, B., Miller, D. L., Voss, M. W., Prakash, R. S., Chaddock, L., ... Wojcicki, T. R. (2012). Caudate nucleus volume mediates the link between cardiorespiratory fitness and cognitive flexibility in older adults. Journal of Aging Research, 2012, 1-11. doi:10.1155/2012/939285

Willingham, D. B., \& Koroshetz, W. J. (1993). Evidence for dissociable motor skills in Huntingtons disease patients. Psychobiology, 21, 173-182.

Wilson, M. A., \& McNaughton, B. L. (1994). Reactivation of hippocampal ensemble memories during sleep. Science, 265, 676-679. doi: $10.1126 /$ science. 8036517 